\title{
Monitoring MVOC Profiles over Time from Isolates of Aspergillus flavus Using SPME GC-MS
}

\author{
Dongdi Sun'1, Alicia Wood-Jones², Wenshuang Wang ${ }^{3}$, Chris Vanlangenberg 3 , David Jones ${ }^{4}$, \\ Julie Gower' ${ }^{1}$ Patrice Simmons ${ }^{1}$, Richard E. Baird ${ }^{2}$, Todd E. Mlsna1* \\ ${ }^{1}$ Department of Chemistry, Mississippi State University, Mississippi State, USA \\ ${ }^{2}$ Department of Biochemistry, Moleculary Biology, Entomology, and Plant Pathology, Mississippi State \\ University, Mississippi State, USA \\ ${ }^{3}$ Department of Mathematics and Statistics, Mississippi State University, Mississippi State, USA \\ ${ }^{4}$ Department of Sustainable Bioproducts, Mississippi State University, Mississippi State, USA \\ Email: ${ }^{*}$ tmlsna@chemistry.msstate.edu
}

Received 26 February 2014; revised 1 April 2014; accepted 10 April 2014

Copyright (C) 2014 by authors and Scientific Research Publishing Inc.

This work is licensed under the Creative Commons Attribution International License (CC BY).

http://creativecommons.org/licenses/by/4.0/

(c) (i) Open Access

\begin{abstract}
Fungi produce a variety of microbial volatile organic compounds (MVOCs) during primary and secondary metabolism. The fungus, Aspergillus flavus, is a human, animal and plant pathogen which produces aflatoxin, one of the most carcinogenic substances known. In this study, MVOCs were analyzed using solid phase microextraction (SPME) combined with GCMS from two genetically different $A$. flavus strains, an aflatoxigenic strain, NRRL 3357, and a non-aflatoxigenic strain, NRRL 21882. A PDMS/CAR SPME fiber was used over 30 days to observe variations in MVOCs over time. The relative percentage of individual chemicals in several chemical classes (alcohols, aldehydes, esters, furans, hydrocarbons, ketones, and organic acids) was shown to change considerably during the varied fungal growth stages. This changing chemical profile reduces the likelihood of finding a single chemical that can be used consistently as a biomarker for fungal strain identification. In our study, discriminant analysis techniques were successfully conducted using all identified and quantified MVOCs enabling discrimination of the two A. flavus strains over the entire 30-day period. This study underscores the potential of using SPME GCMS coupled with multivariate analysis for fungi strain identification.
\end{abstract}

\section{Keywords}

Aspergillus flavus, Discriminant Analysis, Microbial Volatile Organic Compounds, Multivariate Analysis, Solid Phase Microextraction

\footnotetext{
"Corresponding author.
} 


\section{Introduction}

Aflatoxins are polyketide-derived, secondary fungal metabolites and only three Aspergillus species, A. flavus [1], A. nominus [2] and A. parasiticus [3], are known to produce these naturally carcinogenic compounds [4]. The economic impact is immense because fungal mycotoxin contamination is estimated to affect one quarter of the world's food crops (CAST 2003) including maize, cotton and peanuts [4]. Crop losses are estimated to cost between $\$ 1$ and $\$ 1.5$ billion/year in the United States [5]. These losses do not account for livestock losses or the impact on human health or healthcare costs from exposure to the fungi or to the toxins. In order to minimize the safety issue caused by aflatoxins, maximum levels of aflatoxins in many commodities have been set at levels below 20 ppb by most countries [6] [7]. For example, the US Food and Drug Administration (FDA) has set limits of $20 \mathrm{ppb}$ total aflatoxins for interstate commerce of food and $0.5 \mathrm{ppb}$ for milk [8]. The European Commission has set the limits of 15 and $10 \mathrm{ppb}$ for total aflatoxins on groundnuts and dried fruits, respectively [7]. Many methods have been proposed and are in development for the detection of aflatoxins or A. flavus including those that identify the presence of the toxins and those that identify the fungus.

Conventional analytical methods being used for aflatoxin detection are high-performance liquid chromatography (HPLC), gas chromatography mass spectrometry (GC-MS), enzyme linked immune-sorbent assay (ELISA) and multiplex polymerase chain reaction (multiplex PCR) [9]. These methods can be sensitive, inexpensive and give both qualitative and quantitative measurement of aflatoxins, however, initial enrichment or interference/ inhibitor removal is generally required.

Common identification methods for fungi include fluorescence in situ hybridization, DNA array hybridization, and multiplex tandem PCR [10]. However, there are no published aflatoxigenic-specific PCR primers that are able to successfully differentiate aflatoxigenic and non-aflatoxigenic strains. This is an obvious inconvenience in many industrial applications, particularly in the field of maintaining food safety in crops destined for livestock and human consumption. Thus there is an urgent need for a practical, rapid and cost-effective strategy to identify the presence of aflatoxin-producing fungi.

The method described here focuses on identification and quantification of fungal microbial volatile organic compounds (MVOCs) as a means of identifying the fungal presence. The major source of MVOCs produced by organisms such as fungi and bacteria are from primary (synthesis of DNA, amino and fatty acids) and secondary (oxidation of glucose) metabolism [11]. Some MVOCs, such as 3-methyl-1-butanol, 1-octen-3-ol, 3-octanone and sesquiterpenes have been proposed as indicators for fungal species [12]-[14]. Nilsson et al. [15] reported some unique biomarkers (1-octen-3-ol, 3-octanol and several sesquiterpenes) emitted from Penicillium spp. It has been reported that $A$. flavus produces strain-specific volatiles such as 3-methylbutanol, 2-methyl-1-propanol, hexanol, trans-caryophyllene, nonanal and naphthalene [16]. Moreover, several studies have demonstrated that fungal species produce a unique pattern or grouping of MVOCs that can also be used for species identification [17]. Cluster analysis (CA), principle component analysis (PCA) in 2 or 3 dimensional space, and linear discriminant analysis (LDA) have utilized MVOC data to discriminate bacteria at either the species or strain level [18] [19].

Techniques that involve solid phase vapor collection followed by thermal desorption are widely applied in MVOC analysis. Thermal desorption tubes have been used for field sample collection followed by transportation to a lab for analysis [16] [20]. Solid phase microextraction (SPME) has been used to collect and concentrate MVOCs from fungi and bacteria [21]. This technique has the potential to be part of an efficient method for field applications due to its portability and simplicity. The application of SPME in conjunction with GC-MS has been successfully applied to the detection of indoor mold [22] [23], fungal species identification [24] [25], and the diagnosis of foodborne pathogen infection [26] [27].

The focus of this study was 1) to monitor changes in fungal volatile profiles of two strains of A. flavus for 30 days and 2) to develop a method using multivariate analysis for discriminating aflatoxigenic and non-aflatoxigenic A. flavus that is viable over all 30 days of analysis. The general methods represented in this study can be applied to identify other strains and species of fungus.

\section{Material and Methods}

\subsection{Chemical Standards}

Twenty-six reference chemical standards were purchased from several suppliers: 2-heptanone (99\%), 2-heptanol (98\%), hexanal ( $\geq 97 \%)$, 2-methyl-1-butanol ( $\geq 99 \%)$, 3-methyl-1-butanol (98\%), 2-nonanone ( $\geq 99 \%)$, 2-penta- 
nol (98\%), isovaleraldehyde (97\%), 3-octanone ( $\geq 98 \%)$, 2-pentylfuran ( $\geq 97 \%)$, 2-undecanone (98\%), 2-nonanol (99\%), 1-octen-3-ol (98\%), 2-methylbutyric acid (98\%), methyl isobutyrate (99\%), 1, 2, 4, 5-tetramethylbenzene (98\%), 2-octanone (98\%), ethyl acetate (HPLC grade $\geq 99.7 \%)$, 2-heptanone (99\%), octane (98\%) and ethyl isobutyrate (99\%), Fluka Analytical standards, ethyl isovalerate, ethyl butyrate and ethyl proionate were from Sigma-Aldrich (St. Louis, MO). Pentane (98\%) was obtained from Alfa Aesar (Ward Hill, MA).

\subsection{Fungal Sample Preparation}

The aflatoxigenic strains, NRRL 3357 (L-strain; http://www.aspergillusflavus.org/) and NRRL 21882, were provided by the United States Department of Agriculture-Agricultural Research Service, Corn Host Plant Resistance Research Unit, Mississippi State University, Starkville, MS (USDA-ARS-CHPRRU), Mississippi State University, MS. Both fungal strains were cultured on potato dextrose agar (Difco, Sparks, MD), which was prepared by dissolving $39 \mathrm{~g}$ of the powered agar in $1 \mathrm{~L}$ of purified water and autoclaving at $121^{\circ} \mathrm{C}$ for 15 minutes. The fungal spores were then extracted using a $0.02 \%$ Tween 20 solution and then diluted with distilled water to $2 \times 10^{6}$ spores/ml for inoculation. Corn media (2\%) was prepared by mixing $0.6 \mathrm{~g}$ corn grit (Martha White Yellow Corn Meal, Jackson, Tennessee) with $28 \mathrm{ml}$ distilled water. The mixture was then placed in sterile $40-\mathrm{ml}$ glass headspace vials covered with a polypropylene screw cap and PTFE/silicone septum (Sigma-Aldrich, St. Louis, MO). This basal medium was chosen based on preliminary studies performed in this laboratory and studies performed by Demain [28]. The corn media was autoclaved for 1 hour to avoid contamination. Inoculations were performed by adding $10 \mu \mathrm{l}$ of each spore suspension to the cooled $2 \%$ corn media. Fungal growth took placed in $30 \mathrm{ml}$ of the $2 \%$ corn grit liquid media in the capped $40 \mathrm{ml}$ glass vials. The aflatoxigenic and non-aflatoxigenic A. flavus cultures were prepared in five replicates each and four replicates of non-inoculated corn grit liquid media were used as control. Each vial was incubated in the absence of light at $30^{\circ} \mathrm{C}$ followed by MVOC analysis after 1, 3, 6, 10, 20, 24 and 30 days.

\subsection{SPME Fibers Comparison and MVOCs Analysis}

A SPME fiber comparison study was done in order to optimize MVOC collection. Standard solutions of known fungal MVOCs (1-heptanol, 1-hexanol, 1-octen-3-ol, 2-heptanone, 2-methyl-1-butanol, 2-octanone, 3-methyl-1butanol, 3-octanone, ethyl acetate, ethyl butyrate, ethyl isobutyrate, ethyl isovalerate, ethyl propionate, hexanal, methyl isobutyrate, and styrene) were mixed and diluted with dichloromethane to mixture concentrations between $300 \mathrm{ppm}$ to 10,000 ppm. Final concentrations of hydrocarbons (5 ppb), alcohols (300 ppb), ketones (20 $\mathrm{ppb})$, aldehydes (20 ppb), esters (20 ppb) and organic acids (20 ppb) were achieved when $1 \mu \mathrm{l}$ of the standard solutions were injected with a $1 \mu \mathrm{l}$ syringe into $30 \mathrm{ml}$ of deionized water in $40 \mathrm{ml}$ septa equipped vials. SPME fibers with the following materials and thickness were tested: $100 \mu \mathrm{m}$ Polydimethylsiloxane (PDMS), $85 \mu \mathrm{m}$ Carboxen/PDMS (CAR/PDMS), $65 \mu \mathrm{m}$ Divinylbenzene/PDMS (DVB/PDMS), $85 \mu \mathrm{m}$ Polyacrylate (PA) and Carboxen/Divinylbenzene/PDMS (CAR/DVB/PDMS) fibers (Supelco Inc., Bellefonte, PA, USA). The standard volatiles were extracted in triplicate for each type of SPME fiber for one hour at $30^{\circ} \mathrm{C}$.

The CAR/PDMS fiber was selected for headspace extraction of the fungal isolates and non-inoculated corn control for one hour at $30^{\circ} \mathrm{C}$. After 1 hour of exposure the fiber was pulled into the needle sheath, the SPME device was removed from the vial and then inserted into the injection port of GC system for thermal desorption. In order to monitor the changes in VOC profiles from fungal species over time, the VOC metabolites were collected and analyzed after 1, 3, 6, 10, 20, 24 and 30 days.

\subsection{GCMS Conditions}

All GC-MS analysis was performed on an Agilent 5975C Inert XL MSD coupled with 7890A Gas Chromatography system. SPME fibers were desorbed at $250^{\circ} \mathrm{C}$ in a split/splitless injection port, equipped with a $78.5 \mathrm{~mm}$ $\times 6.5 \mathrm{~mm} \times 0.75 \mathrm{~mm}$ SPME inlet liner (Supelco Inc., Bellefonte, PA, USA) while working in the splitless mode. The GC system was equipped with a DB-1 capillary column $(60 \mathrm{~m} \times 320 \mu \mathrm{m} \times 1 \mu \mathrm{m})$. Helium was used as a carrier gas with a flow velocity of $1.2 \mathrm{ml} \cdot \mathrm{min}^{-1}$. The oven temperature program was as follows: $45^{\circ} \mathrm{C}$ held for 9 min, $10^{\circ} \mathrm{C} \cdot \mathrm{min}^{-1}$ ramp to $85^{\circ} \mathrm{C}$ followed by a $3 \mathrm{~min}$ hold; ramp to $120^{\circ} \mathrm{C}$ at $3^{\circ} \mathrm{C} \cdot \mathrm{min}^{-1}$ followed by a $3 \mathrm{~min}$ hold, then a final ramp at $10^{\circ} \mathrm{C} \cdot \mathrm{min}^{-1}$ to $270^{\circ} \mathrm{C}$. The MS analysis was carried out in full scan mode (scan range from $35-350 \mathrm{amu}$ ) with ionization energy of $70 \mathrm{eV}$. Ion source and quadrupole temperatures were $230^{\circ} \mathrm{C}$ and $150^{\circ} \mathrm{C}$, 
respectively. Fungal metabolites were identified by comparing the retention time of chromatographic peaks with standards analyzed under the same conditions and by mass spectrum database search using the NIST 08 spectral database.

\subsection{Multivariate Analysis}

Discriminant analysis (DA) was employed to visualize resultant clustering of fungal culture samples based on MVOC profiles and to examine the relationship between toxigenic and non-toxigenic A. flavus isolates. Prior to analysis, peak area data were standardized (Z-score) to mean zero and unit variance. The signal zero mean was calculated by removing the average and the unit variance by dividing the standard deviation. Discriminant analysis was performed using statistic software IBM SPSS statistics 21 (International Business Machines Corp.).

\section{Results and Discussion}

\subsection{HS-SPME Extraction Method Optimization}

To investigate the extraction efficiency for the MVOCs, the following specific fibers were evaluated: $100 \mu \mathrm{m}$ PDMS, $85 \mu \mathrm{m}$ CAR/PDMS, $65 \mu \mathrm{m}$ DVB/PDMS, $85 \mu \mathrm{m}$ PA and 50/30 $\mu \mathrm{m}$ CAR/DVB/PDMS. Figure 1 shows the resulting TIC chromatograms for the 17 standard VOC mixture after one hour headspace extraction at $30^{\circ} \mathrm{C}$ (best temperature for aflatoxin production). The data is displayed on the same scale to emphasize the difference in extraction efficiencies. PDMS and PA fibers were determined to be not suitable because of relatively low collection amounts when compared to the other fiber types. CAR/PDMS, DVB/PDMS and CAR/DVB/PDMS fibers show similar TIC chromatograms. For further investigation, the peak areas of the 17 standard VOCs obtained by the three types of fibers were compared as shown in Figure 2. The average relative standard deviations of the 17 standard VOCs for these fibers are 18.4\% (CAR/PDMS), $13.1 \%$ (DVB/PDMS) and 14.9\% (CAR/DVB/PDMS). Although DVB coated fibers extracted larger amount of high molecular weight alcohols and ketones (1-octen-3-ol, 2-octanone and 3-octanone), they have less affinity to esters (ethyl butyrate, ethyl isobutyrate and methyl isobutyrate) and low molecular weight alcohols (3-methyl-1-butanol and 2-methyl-1butanol). Furthermore, insufficient amounts of 2-methyl-1-propanol and ethyl acetate were collected using DVB coated fibers to permit detection; therefore, CAR/PDMS fiber was used in the subsequent fungus MVOC studies. A culture media volume of $30 \mathrm{~mL}$ and $10 \mathrm{~mL}$ headspace volume provided sufficient amounts of VOCs during a 1 hour collection period at $30^{\circ} \mathrm{C}$. The choice of SPME fiber for MVOCs collection technique plays an important role in fungal species discriminations. It should be noted that SPME fiber sample collection is an attractive option for field analysis when combined with portable detector devices [29]. For example, CAR/PDMS fibers are better for volatile analytes while DVB/PDMS fibers are good for extracting semi-volatile analytes. The CAR/ DVB/PDMS fiber contains two adsorbents and can extend the molecular weight range of analytes extracted with a single SPME fiber. However, in this study, the CAR/PDMS fiber was selected because of our desire to focus on collection of the more abundant volatile organic compounds being emitted from the two fungal strains.

\subsection{Identification of Volatiles Produced by A. flavus}

The volatile MVOC profiles produced by aflatoxigenic and non-aflatoxigenic $A$. flavus were monitored over 30 days. The resulting chromatograms obtained from the headspace analysis of the emitted MVOCs after incubation for 6 days are shown in Figure 3 for the control (growth media only), toxic (aflatoxigenic A. flavus) and nontoxic (non-aflatoxigenic A. flavus) samples. A very clear difference in MVOCs abundance was observed where the toxic strain produces significantly less MVOCs compared to the nontoxic strain. MVOCs produced by the fungal strains and control were identified by comparing with the standards and the NIST 08 library. Ethanol was produced in significantly large amounts in all fungal cultures; we found that this chemical did not aid in discrimination and was therefore removed from consideration when looking for identifying MVOCs patterns. The most significant signals (detected in all replicates) with high abundance (TIC peak area $>1 \times 10^{4}$ units) are listed in Table 1 (excluding ethanol). This table contains the chemical retention time, standard deviation of this retention, compound name, the days the specific chemical was detected in the samples and its relative composition. 
Table 1. Headspace SPME-GCMS analysis of 52 microbial volatile metabolites from both aflatoxigenic and non-aflatoxigenic strains of Aspergillus flavus.

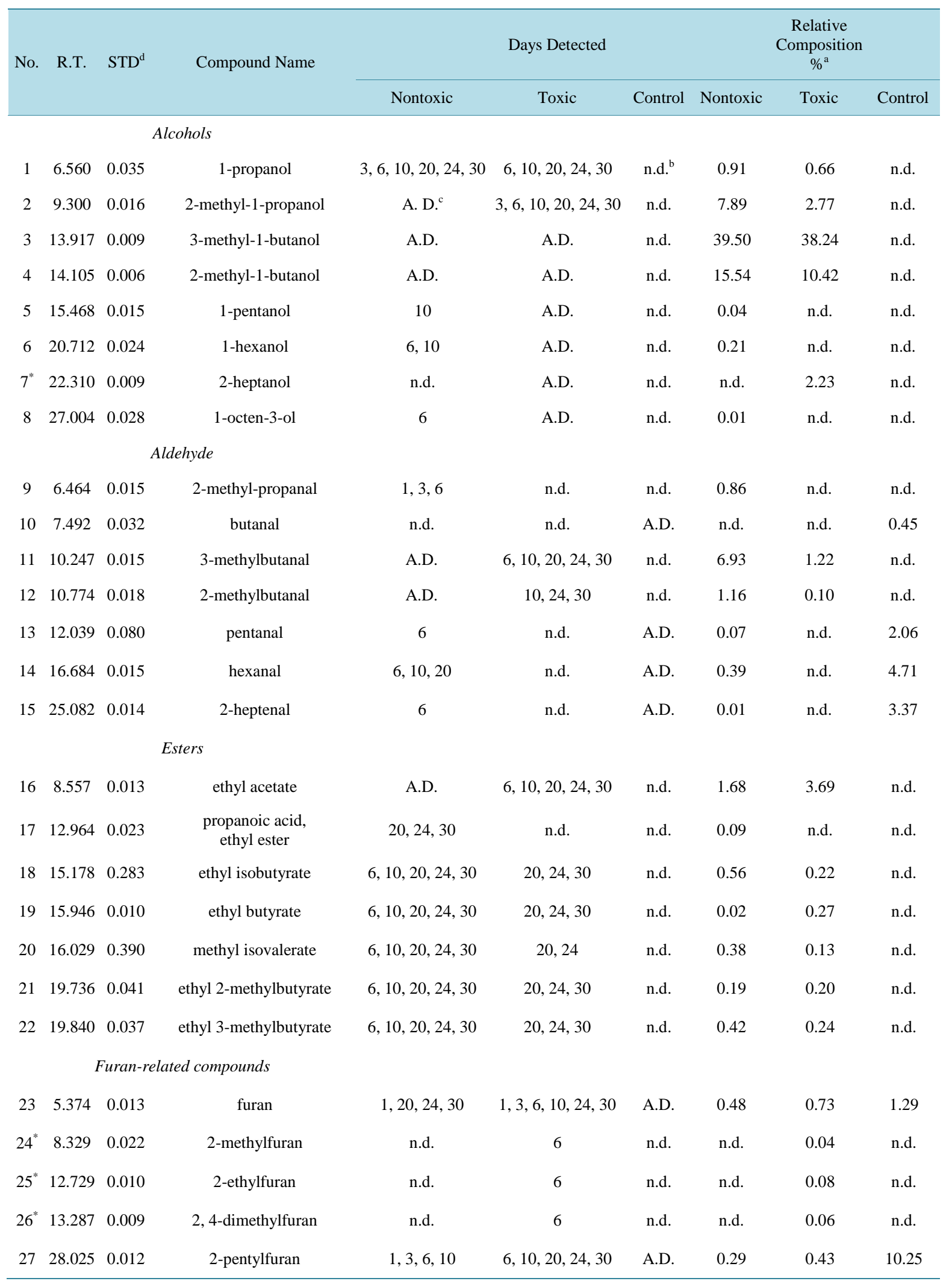




\section{Continued}

\begin{tabular}{|c|c|c|c|c|c|c|c|c|c|}
\hline \multicolumn{10}{|c|}{ Hydrocarbons } \\
\hline 28 & 5.535 & 0.011 & pentane & A.D. & A.D. & A.D. & 6.94 & 11.45 & 36.51 \\
\hline 29 & 7.321 & 0.015 & 2-methylpentane & n.d. & 6,10 & n.d. & n.d. & 0.26 & 0.52 \\
\hline 30 & 8.544 & 0.015 & hexane & n.d. & n.d. & A.D. & n.d. & n.d. & 1.05 \\
\hline 31 & 12.684 & 0.007 & 2, 2, 3, 3-tetramethylbutane & 1,3 & 1,3 & A.D. & 1.78 & 0.41 & 8.61 \\
\hline 32 & 13.061 & 0.060 & heptane & 6,24 & 6 & A.D. & 0.08 & 0.12 & 3.87 \\
\hline 33 & 14.463 & 0.010 & 2, 5-dimethylhexane & 1,3 & n.d. & A.D. & 0.19 & n.d. & 0.73 \\
\hline 34 & 14.583 & 0.012 & 2, 4-dimethylhexane & 1,3 & n.d. & A.D. & 0.61 & n.d. & 2.49 \\
\hline 35 & 15.371 & 0.007 & 2, 3, 4-trimethylPentane & 1,3 & 1,3 & A.D. & 1.48 & 0.43 & 5.06 \\
\hline 36 & 15.609 & 0.008 & 2, 3, 3-trimethylPentane & 1,3 & 1,3 & A.D. & 1.80 & 0.68 & 4.01 \\
\hline 37 & 15.681 & 0.138 & toluene & $6,10,20,24$ & n.d. & n.d. & 0.33 & n.d. & n.d. \\
\hline 38 & 15.843 & 0.005 & 2, 3-dimethylhexane & 1,3 & 3 & A.D. & 0.42 & 0.09 & 1.62 \\
\hline 39 & 17.826 & 0.009 & octane & 6,30 & 6,24 & A.D. & 0.06 & 0.22 & 4.64 \\
\hline 40 & 21.284 & 0.024 & 2, 3, 4-trimethylhexane & 1 & 6 & A.D. & 0.05 & 0.05 & 0.48 \\
\hline 41 & 22.085 & 0.027 & styrene & 6,10 & n.d. & n.d. & 0.14 & n.d. & n.d. \\
\hline 42 & 22.367 & 0.007 & p-xylene & $3,20,24$ & n.d. & n.d. & 0.09 & n.d. & n.d. \\
\hline 43 & 25.295 & 0.016 & $\alpha$-pinene & $6,10,20,24,30$ & n.d. & n.d. & 0.25 & n.d. & n.d. \\
\hline 44 & 35.812 & 0.007 & decane & 3 & n.d. & n.d. & 0.20 & n.d. & 0.20 \\
\hline \multicolumn{10}{|c|}{ Ketones } \\
\hline 45 & 5.126 & 0.017 & acetone & A.D. & A.D. & A.D. & 1.19 & 1.24 & 1.22 \\
\hline 46 & 7.263 & 0.041 & 2, 3-butanedione & $3,6,10,14,20,24$ & $20,24,30$ & n.d. & 0.58 & 0.11 & n.d. \\
\hline 47 & 11.758 & 0.012 & 2-pentanone & $1,3,10,20,24,30$ & $1,3,24,30$ & n.d. & 0.39 & 0.45 & n.d. \\
\hline 48 & 12.375 & 0.022 & 3-hydroxy-2-butanone & $3,6,10,20,24,30$ & n.d. & n.d. & 0.37 & n.d. & n.d. \\
\hline 49 & 21.486 & 0.006 & 2-heptanone & 1,3 & A.D. & A.D. & 2.36 & 17.67 & 1.21 \\
\hline 50 & 27.147 & 0.005 & 3-octanone & 6 & $1,20,24,30$ & n.d. & 0.01 & 0.16 & n.d. \\
\hline 51 & 27.341 & 0.014 & 2-octanone & 1 & $1,6,10,20,24,30$ & A.D. & 0.13 & 0.36 & 0.24 \\
\hline 52 & 34.108 & 0.005 & 2-nonanone & 1 & $1,3,6$ & n.d. & 0.30 & 3.28 & n.d. \\
\hline \multicolumn{10}{|c|}{ Organic acids } \\
\hline 53 & 7.463 & 0.179 & acetic acid & $10,20,24,30$ & $6,10,20,24,30$ & n.d. & 1.52 & 1.08 & n.d. \\
\hline 54 & 14.828 & 0.172 & 2-methylpropanoic acid & $6,10,20,24,30$ & 30 & n.d. & 0.72 & 0.16 & n.d. \\
\hline 55 & 19.575 & 0.169 & 2-methylbutanoic acid & $6,10,20,24,30$ & n.d. & n.d. & 0.36 & n.d. & n.d. \\
\hline \multicolumn{10}{|c|}{ Sulfur containing compounds } \\
\hline $56^{*}$ & 5.740 & 0.004 & dimethyl sulfide & n.d. & 3 & n.d. & n.d. & 0.07 & n.d. \\
\hline
\end{tabular}

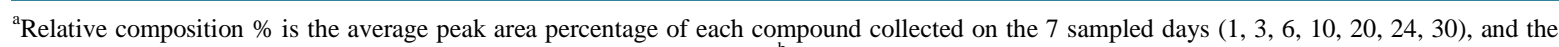
peak area of each compound for days not detected was counted as zero; ${ }^{b}$ n.d.: not detected in the culture samples which were analyzed by GCMS; ${ }^{\mathrm{c}}$ A.D.: detected in all days sampled (1, 3, 6, 10, 20,2 4, 30); ${ }^{\mathrm{d}}$ STD: standard deviation of each compound retention time in five replicates; ${ }^{\mathrm{e}}$ Identification based on the comparison of retention time and mass spectra with standards under the same conditions; ${ }^{{ }^{*}}$ : VOCs detected in aflatoxigenic $A$. flavus only; ${ }^{\mathrm{g}} \mathrm{Ethanol}$ and carbon dioxide was detected in all samples; it is not listed due to large amount of VOC production interference the other peak area \% result. 

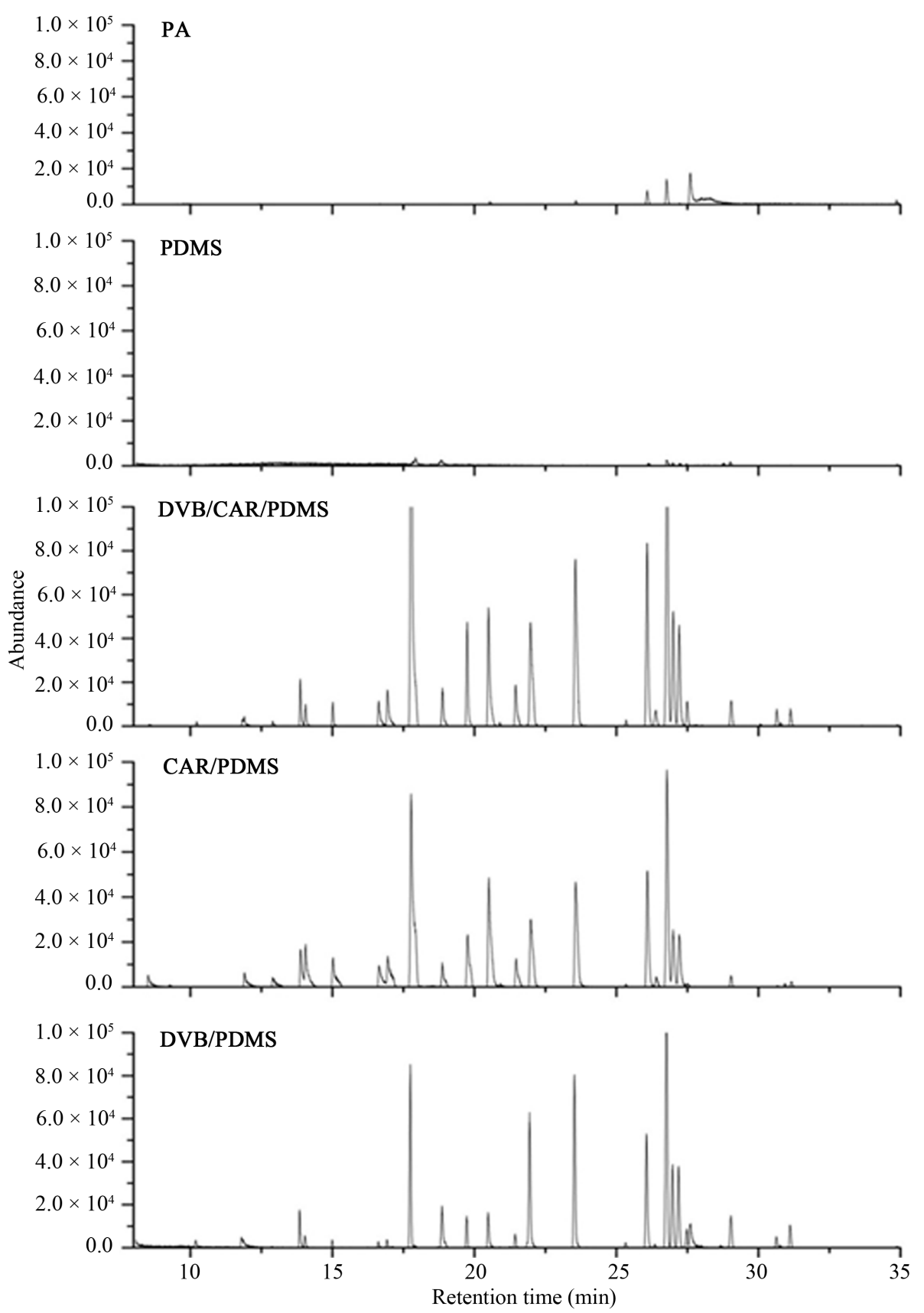

Figure 1. Comparison of TIC chromatograms from varied SPME extraction of 17 standard VOCs followed by GCMS analysis displayed on the same scale. Best results were obtained using DVB/CAR/PDMS, DVB/PDMS and CAR/PDMS.

In Table 1, the relative composite percentage of each compound shows the average peak area percentage of the listed MVOCs during the 30 days of incubation. The detected MVOCs were further clustered by functional group including alcohols, aldehydes, esters, furans, hydrocarbons, ketones, and organic acids. In total, 57 different volatile compounds were identified in all samples (fungus and control). Twenty-seven compounds were detected in the non-aflatoxigenic strain, and 25 compounds were detected in the aflatoxigenic strain. The predominant MVOCs were alcohols (ethanol, 2-methyl-1-propanol, 3-methyl-1-butanol, and 2-methyl-1-butanol), aldehydes (3-methylbutanal, 2-methylbutanal), esters (ethyl isobutyrate, methyl isovalerate), hydrocarbons (toluene, $\alpha$-pinene, and styrene), ketones (2, 3-butanedione, 3-octanone) and organic acids (acetic acid, 2-methyl- 


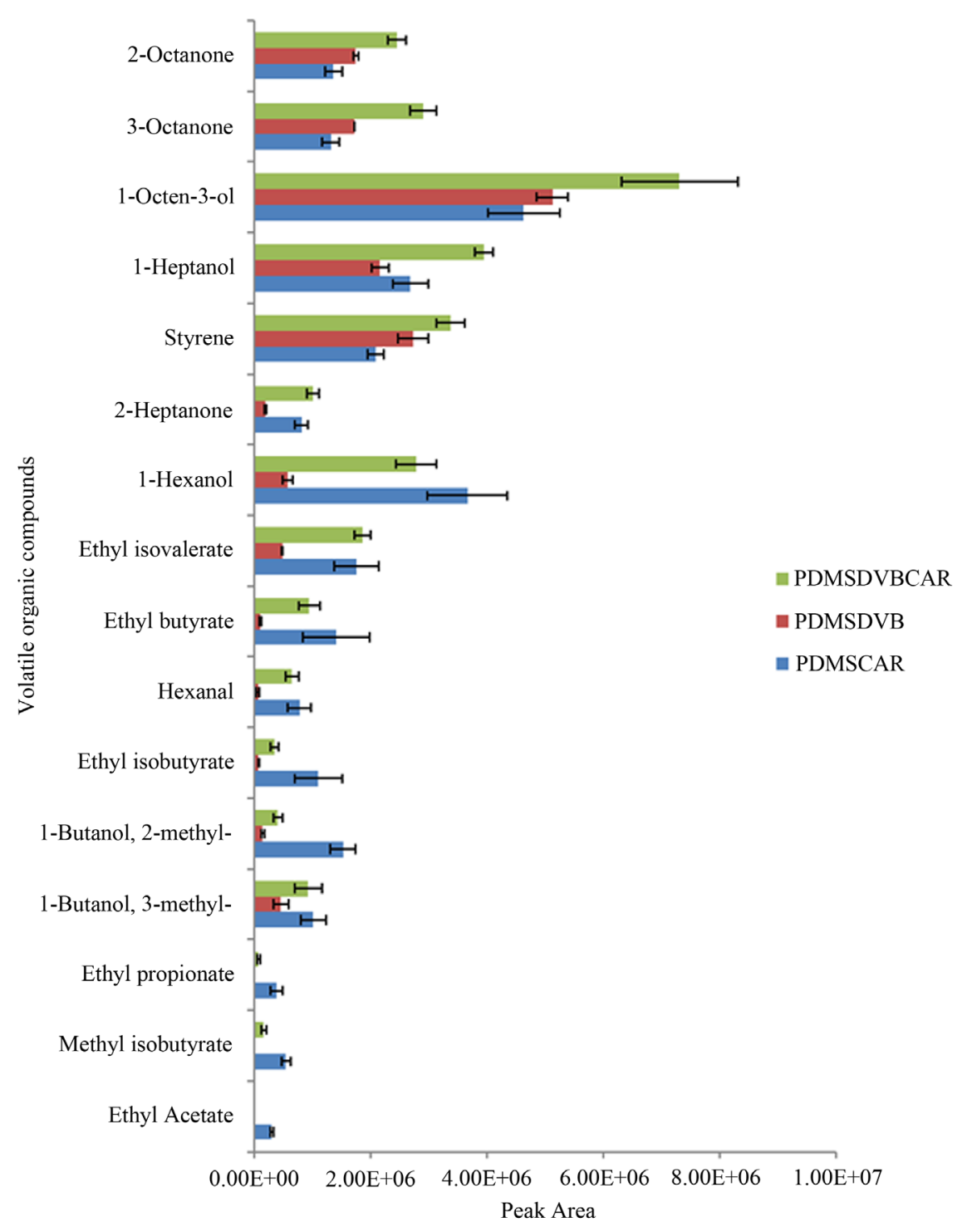

Figure 2. Comparison of peak areas showing 17 standard VOCs after HS-SPME-GCMS analysis using different SPME fiber coating, including CAR/DVB/PDMS, DVB/PDMS and CAR/PDMS. Each fiber was tested in triplicate.

propanoic acid). Alcohols and esters were found in the fungal culture samples and were not detected in the corn control media. In addition, most of the hydrocarbons were produced by the corn control; however some hydrocarbons (toluene, styrene and $\alpha$-pinene) were only emitted by the non-aflatoxigenic strain. A relatively high percentage of 2-heptanol (2.23\%) consistently appeared in volatiles produced by the aflatoxigenic strain however this compound was not found in the non-aflatoxigenic strain. Moreover, a low percentage of furans (2-methylfuran, 2-ethylfuran, and 2, 4-dimethylfuran) were detected at day 6 and dimethyl sulfide was detected at day 3 only in aflatoxigenic strain.

Several observations can be made from Table 1 data. Significant amounts of ethanol were formed from the metabolic oxidation of glucose during the primary and the secondary metabolism of non-aflatoxigenic and aflatoxigenic A. flavus cultures. Ethanol was also observed by Jurjevic et al. [16] in the headspace gases produced by the aflatoxigenic and non-aflatoxigenic strains grown on the corn substrate for 25 days incubation. Several MVOCs were found in our study on most days in both the non-aflatoxigenic and aflatoxigenic strains including 3-methyl-1-butanol, 2-methyl-1-butanol, 2-methyl-1-propanol and 3-octanone. No single chemical was unique to a specific fungi strain. 

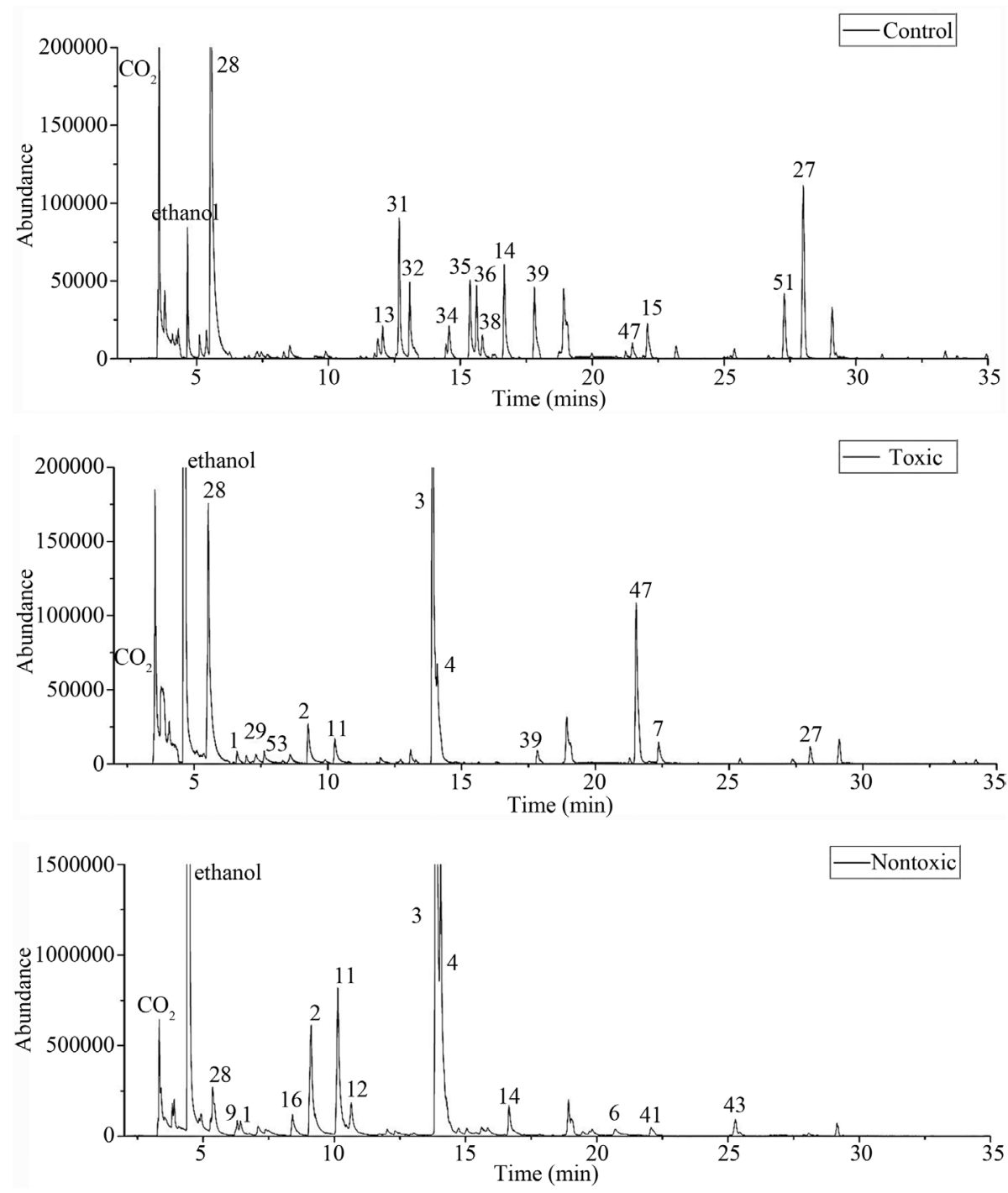

Figure 3. HS-SPME-GCMS total ion current (TIC) chromatogram showing MVOCs identified from the fungal strains and non-inoculated media at day 6 for the control (upper), toxigenic A. flavus (center), and non-toxigenic A. flavus (lower). Peak numbers refer to the volatiles listed in Table 1. (Ethanol and carbon dioxide was detected in all samples).

Certain MVOCs produced by A. flavus have been reported to be biomarkers that can be used for identifying pathogenic fungal strains. For example, $\mathrm{C}_{15} \mathrm{H}_{24}$ volatile compounds (alpha-gurjunene, trans-caryophyllene, and cadinene) were detected using a purge and trap technique and were considered to be unique "fingerprints" for aflatoxigenic strains of A. flavus [30]. Our analysis did not observe these chemicals most likely because the low volatility of these compounds is not conducive to SPME collection in the presence of more volatile and prevalent chemicals.

Dimethyl disulfide and nonanal were reported to be associated only with the aflatoxigenic A. flavus, while hexanal, 1-hexanol, 1-octen-3-ol, and 2-pentyl furan were only associated with non-toxigenic A. flavus [16]. Our study also identified several compounds that were found in only one isolate. However, these compounds cannot be used for discrimination because this trend did not hold up over time during each lifecycle stage. For example in agreement with the literature we found dimethyl disulfide only in our toxic sample but this chemical was present only on the $3^{\text {rd }}$ day, while 1-hexanol, propyl ethyl ester, and 2-methylbutanoic acid where only found in the nontoxic A. flavus but again these chemicals were not present on each day. One exception was 1-heptanol, this chemical was present in all toxic samples but was not found in any of the non-toxic samples. 


\subsection{Investigation of the Fungal VOC Profile over Time}

Variations in MVOCs patterns over time were determined using peak area percentage utilizing mass spectrum total ion counts. The total amounts of MVOCs from aflatoxigenic and non-aflatoxigenic strains were investigated during 30 days incubation as shown in Figure 4. Total peak areas for each day were calculated by summing the peak areas of all detected MVOCs in a sample (excluding ethanol). The results show that the amount of MVOCs significantly increases by day 6 for the nontoxic isolate relative to the toxic isolate due primarily to increasing amounts of alcohols and esters being produced. It is interesting to note that, after 10 days the quantity of MVOCs begins to decrease, possibly because a lack of nutrients remaining in the media retards the biosynthetic process of fungi. The results found in Figure 4 which shows that non-aflatoxigenic and aflatoxigenic $A$. flavus produce significantly different amount of MVOCs over 30 days. We hypothesize that the difference in amount of MVOCs production are caused by the following reasons: 1) Aflatoxin biosynthesis is induced by simple carbohydrates, such as glucose and sucrose [31], therefore aflatoxin production reduces nutrients available for fungi growth; 2) The non-toxigenic isolate has a characteristic gene for rapid growth compared to toxigenic isolate; 3) The presence of aflatoxin inhibits some biological pathways that produce MVOCs.

Figure 5 shows time-dependent expression patterns of six chemical classes in defined time intervals. The (non-ethanol) alcohol production (mainly 3-methyl-1-butanol, 2-methyl-1-butanol and 2-methyl-1-propanol) significantly increased during incubation and reached maximum at about 20 - 24 days (Figure 5(a)). In the late period of incubation (20 - 30 days), the relative percentages of esters are much higher in the aflatoxigenic strain compared to non-aflatoxigenic strain (Figure 5(c)). The production of esters (ethyl isobutyrate, methyl isovalerate and ethyl 3-methylbutyrate) and organic acids (acetic acid and 2-methylpropanoic acid) increased significantly starting on day 6 with another significant increase for the ester in the toxic isolate beginning on day 20. However, the relative percentage of aldehydes and ketones decreased during the 30 days of fungal culture incubations. The large percentage of ketones produced by the aflatoxigenic strain in the early stage of incubation (Figure 5(c)) is primarily from 2-heptanone production. Thus, we can report that even though some trends are observed we see significant variations in MVOCs production over time.

Our results demonstrate that there are numerous qualitative and quantitative fluctuations in MVOCs profiles during different days as shown in Table 1 and Figure 5 consistent with the findings of Borjesson et al. [32] and Jurjevic et al. [16]. A significant distinction in the relative amounts of MVOCs production from aflatoxigenic and non-aflatoxigenic A. flavus provides a possible direction for discriminating fungal. However, developing a method that discriminates on a specific growth day is not applicable for field analysis, since the growth stage of fungal species cannot be ascertained when collecting MVOCs in the field. Korpi et al. [11] also emphasized that

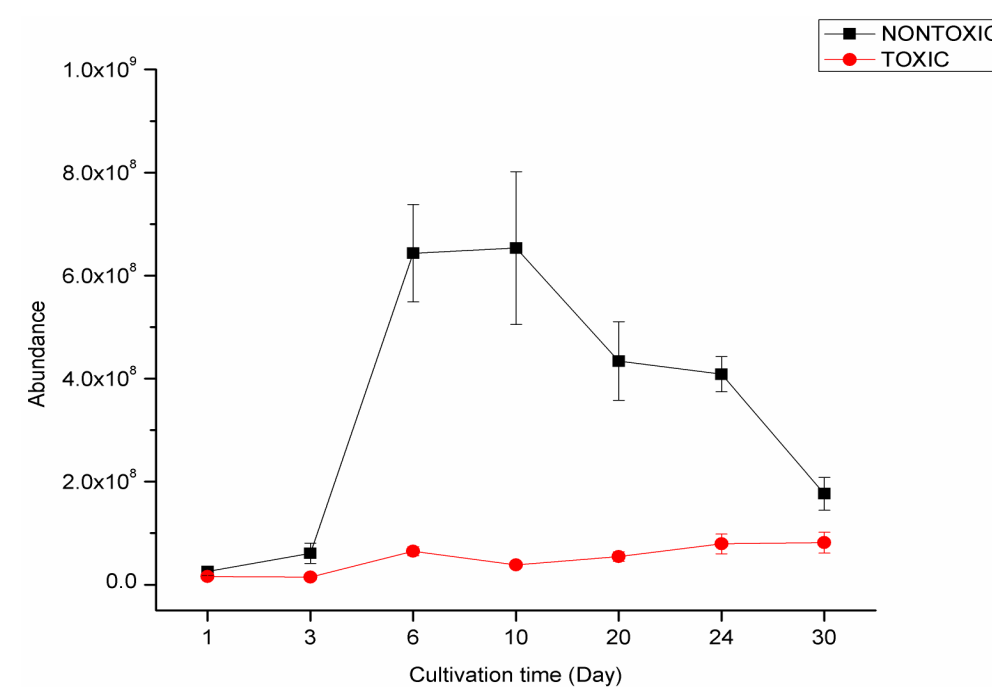

Figure 4. Comparison of total amount of MVOCs between aflatoxigenic and non-aflatoxigenic $A$. flavus during a cultivation period of 30 days. The abundance is the total peak area of all compounds detected from both aflatoxigenic and non-toxigenic $A$. flavus. 


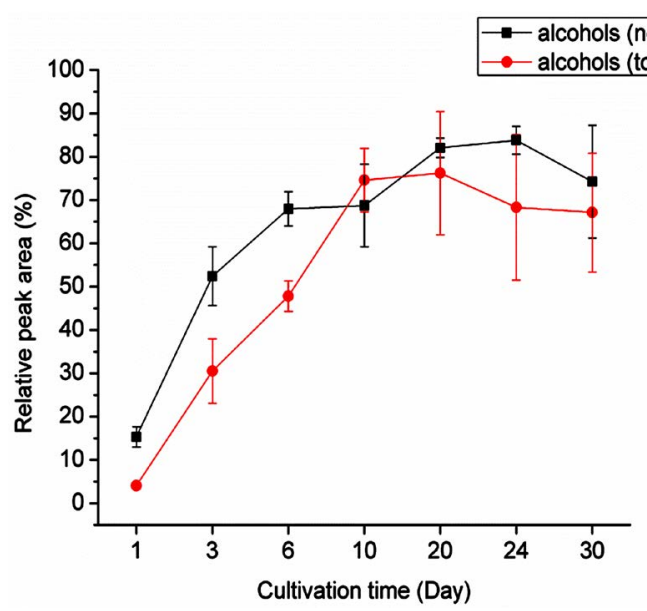

(a)

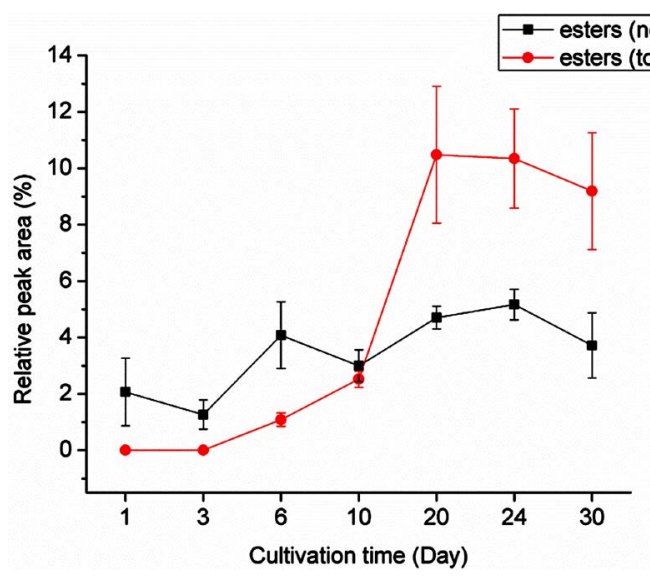

(c)

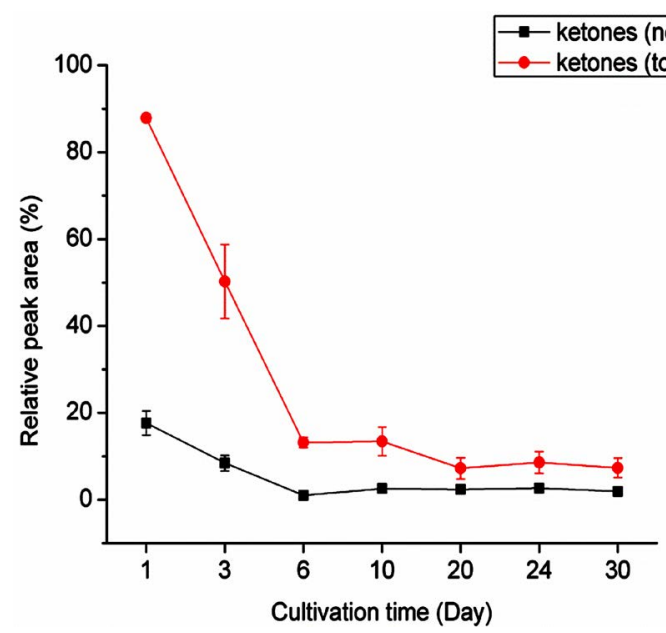

(e)

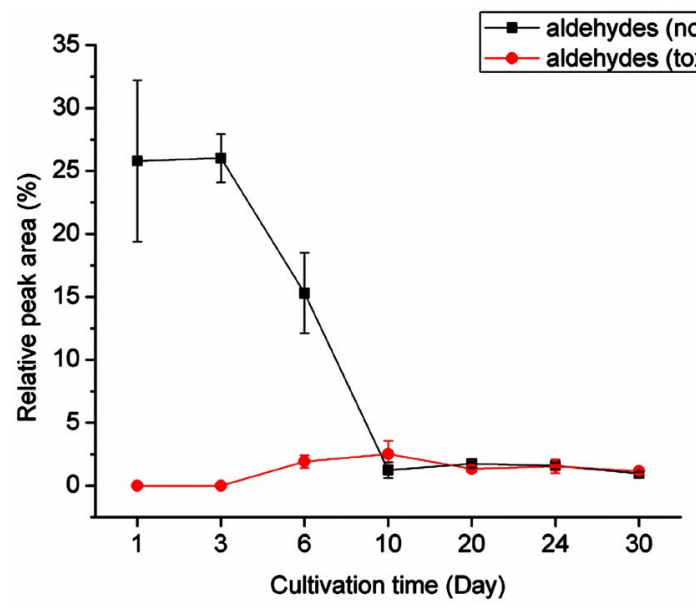

(b)

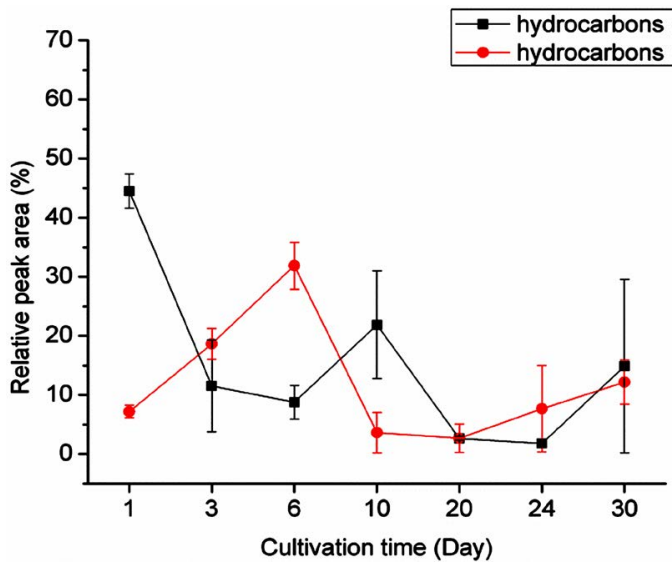

(d)

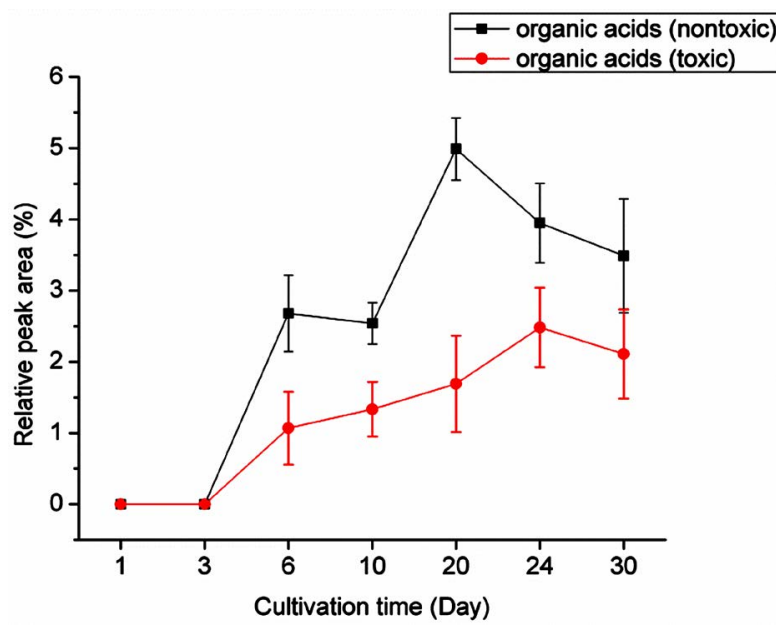

(f)

Figure 5. Variation of MVOCs expression patterns of aflatoxigenic and non-aflatoxigenic A. flavus during a cultivation period of 30 days for selected volatiles from classified compounds of a) alcohols; b) aldehydes; c) esters; d) hydrocarbons; e) ketones; and f) organic acids.

an individual MVOC cannot be related to a certain microbial species because the same MVOC may be produced by different microorganisms. In order to reduce the reliance on any one specific MVOC we have applied che- 
motaxonomy techniques to reveal potential species-specific MVOC patterns by comparing entire qualitative and quantitative datasets of MVOCs. More importantly, this method enables the discrimination of fungal strains during any growth stage during the first 30 days.

\subsection{Multivariate Analysis of MVOC Profile}

Due to the large number and varying concentrations of MVOCs produced, multivariate analysis is required to recognize patterns in the data leading to discrimination of the different fungal strains. To evaluate the capability of this HS-SPME-GCMS method for distinguishing aflatoxigenic and non-aflatoxigenic A. flavus, the GCMS data (day 1, 3, 6, 10, 20, 24, 30) from fungi and control samples were collected and analyzed using discriminant analysis (DA) models. DA builds up a predictive model which is composed of a discriminant function based on linear combinations of predictor variables. It can be used to discard variables that are little related to group distinctions and to maximally separate the groups. Using this approach, 13 MVOCs (Table 2) were identified with 2-methyl-1-propanol, 2-heptanol, propanoic acid ethyl ester, ethyl isobutyrate, ethyl 3-methylbutyrate, furan, 2pentylfuran, 2, 3-butanedione, 2-heptanone, 2-octanone, and 2-methylpropanoic being the most significant compounds for group classification. The relative peak area percentage of these compounds (three groups) has significant change during the 30 days incubation as shown in Figure 6. Figure 7 shows the plot of discriminant scores of the analyzed samples. The three classified groups (toxic, nontoxic, control) were satisfactorily separated, showing that this method can be used to discriminate these strains of aflatoxigenic and non-aflatoxigenic A. flavus during the fungi growing process. All of the group cases were correctly classified by the discriminant functions built by the model, thus achieving perfect discrimination (Table 3).

Multivariate analysis was performed by utilizing the standardized data for each identified compound produced by control and fungal strains to discriminate aflatoxigenic and non-aflatoxigenic strains. Multivariate analysis is a powerful technique for this sort of complex data because it can reveal hidden patterns and reduce the information to a more comprehensive format [31]. In this study, discriminate analysis was used, unlike principle component analysis and cluster analysis, object groups are known in discriminate analysis and the goal is to determine the best fit parameters of the model to separate the objectives base on independent variables of samples. In this case the categorical groups are aflatoxigenic and non-aflatoxigenic strains, as well as the corn control. The independent variables used for discrimination are qualitative (compound name) and quantitative (standardized peak area). DA was applied to calculate the discrimination functions for classification of aflatoxigenic, non-af-

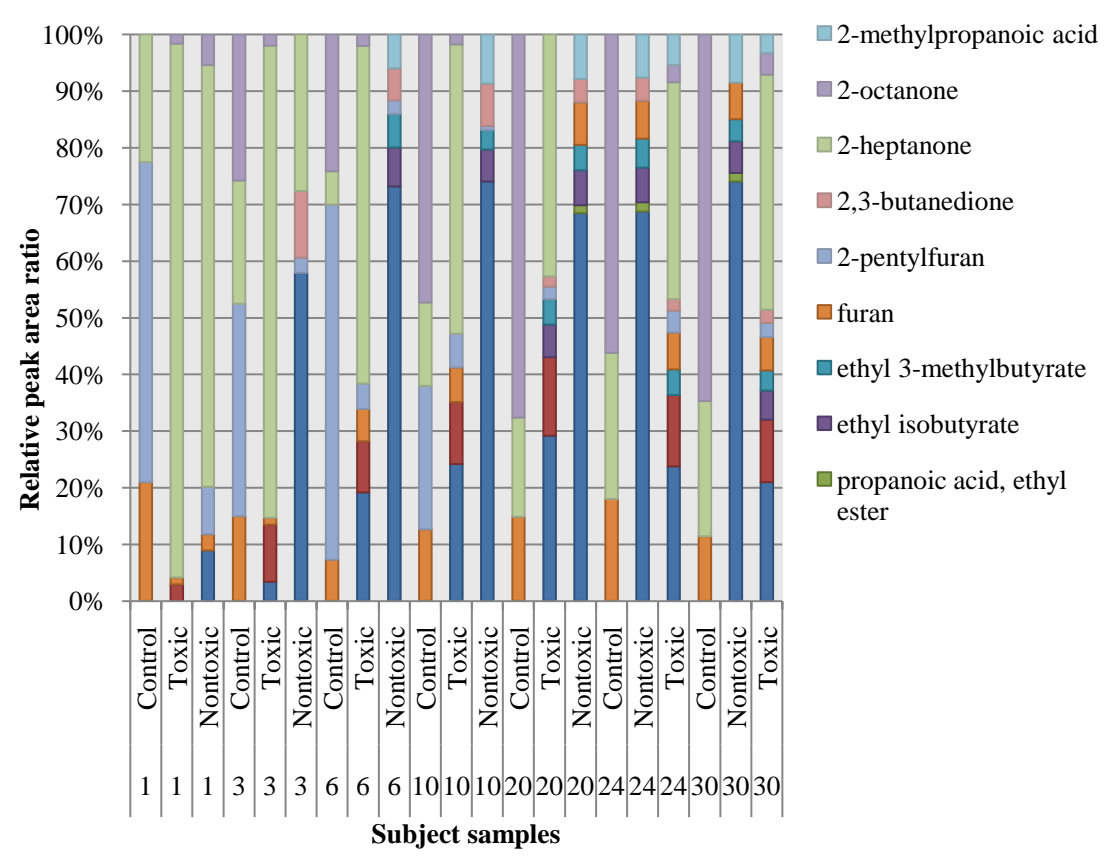

Figure 6. Color stacked bar chart of the 11 most significant MVOCs (relative percent). A visual representation of the data used for discriminant analysis. 


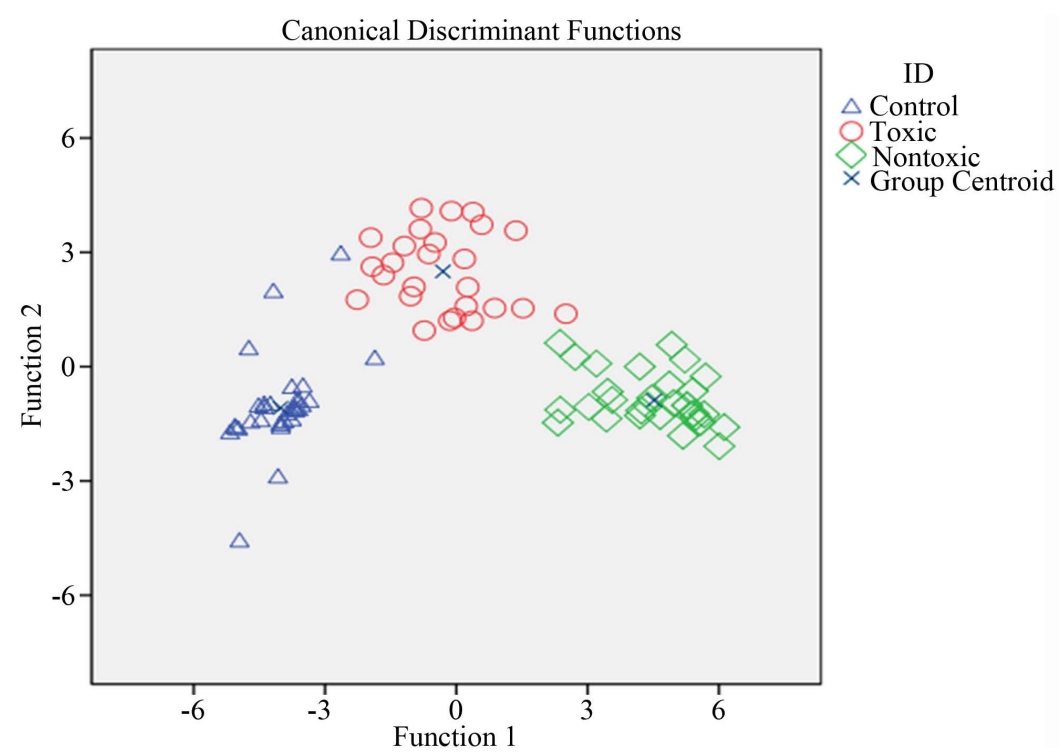

Figure 7. Discriminant score plot of the MVOCs analyzed by HS-SPME-GCMS grouped by chemical classes of toxigenic and non-toxigenic isolates and non-inoculated control during 30 days incubation.

Table 2. Standardized canonical discriminant function coefficients for HS-SPME-GC-MS data from samples analyzed during 30 days culture incubation.

\begin{tabular}{ccc}
\hline Standardized Canonical Discriminant Function Coefficients ${ }^{\mathrm{a}}$ & \\
Variable & \multicolumn{1}{c}{ Discriminant Function $^{\mathrm{b}}$} \\
\hline 2-methyl-1-propanol & 1 & 2 \\
2-heptanol & -1.016 & 0.338 \\
propanoic acid, ethyl ester & -3.056 & -3.086 \\
ethyl isobutyrate & 1.051 & 0.240 \\
2-pentylfuran & -0.109 & -1.219 \\
ethyl, 3-methylbutyrate & 1.889 & 1.535 \\
furan & -1.066 & -0.723 \\
2, 3-dimethylhexane & 0.954 & 1.664 \\
styrene & 1.787 & 1.672 \\
2-octanone & -0.552 & -1.071 \\
2-heptanone & 1.010 & 0.634 \\
2, 3-butanedione & 1.479 & 0.531 \\
2-methylpropanoic acid & 1.450 & 2.008 \\
\hline
\end{tabular}

${ }^{a}$ Discriminant analysis was performed using standardized GC-MS data from aflatoxigenic, non-aflatoxignic $A$ flavus and control samples analyzed in day $1,3,6,10,20,24,30$; ${ }^{\mathrm{b}}$ Discriminant function 1 and 2 were used as linear combinations of independent variables for 3-group discriminant analysis. 
Table 3. Classification and cross-validation results using HS-SPME-GC-MS data from samples analyzed during 30 days culture incubation.

\begin{tabular}{|c|c|c|c|c|c|}
\hline & \multicolumn{5}{|c|}{ Classification Results $^{\mathrm{a}, \mathrm{c}}$} \\
\hline & \multicolumn{4}{|c|}{ Predicted Group Membership ${ }^{\mathrm{d}}$} & \multirow{2}{*}{ Total } \\
\hline & ID & 0 & 1 & 2 & \\
\hline \multirow{3}{*}{ Original } & Control & $97.1 \%$ & $2.9 \%$ & 0 & $100 \%$ \\
\hline & Toxic & 0 & $100 \%$ & 0 & $100 \%$ \\
\hline & Nontoxic & 0 & 0 & $100 \%$ & $100 \%$ \\
\hline \multirow{3}{*}{ Cross-validated $^{\mathrm{b}}$} & Control & $91.2 \%$ & 8.8 & 0 & $100 \%$ \\
\hline & Toxic & 7.7 & $88.5 \%$ & 3.8 & $100 \%$ \\
\hline & Nontoxic & 0 & 0 & $100 \%$ & $100 \%$ \\
\hline
\end{tabular}

${ }^{\mathrm{a}} 98.9 \%$ of original grouped cases correctly classified; ${ }^{\mathrm{b}} \mathrm{Cross}$ validation is done only for those cases in the analysis. In cross validation, each case is classified by the functions derived from all cases other than that case; ${ }^{\circ} 93.5 \%$ of cross-validated grouped cases correctly classified; ${ }^{\mathrm{d}}$ Predicted group membership includes non-inoculated control, non-aflatoxigenic strain culture, aflatoxigenic strain culture.

latoxigenic A. flavus and control in clusters, which minimizes the variance within the classes and maximizes the variance among the classes. DA provides a number of discriminant functions equal to the number of categories of grouping variables minus one. Since three categories were considered including toxic, nontoxic and control, two discriminant functions were obtained in which the first function maximizes the difference between the values of the dependent variables, and the second function. Two discriminant functions were calculated, with the first accounting for $84.1 \%$ of the variance. In summary, the low Wilks' lambda values of function $1(0.019)$ and function 2 (0.282) indicate the ideal discriminatory ability of the functions. The standardized discriminant function coefficients indicate the relative importance of the independent variables in predicting the dependence, where coefficients with large absolute values correspond to variables with greater discriminating ability. A stepwise method was performed by automatically selecting the best MVOCs to use in this model. The "leaveone-out" cross-validation method was performed in order to determine the accuracy of the predictive model, where each identity tested is removed one-at-a-time from the initial matrix of data; then the classification model is rebuilt and the case removed is classified in this new model. The discriminant analysis model based on MVOCs of inoculated samples correctly classified $93.5 \%$ of the observations based on cross-validation. The result obtained from DA can be considered very satisfactory for the detection of aflatoxin producing A. flavus growing in corn media.

\section{Conclusions}

Our results clearly show that the production of MVOCs is significantly affected by microbial species and growth cycles, and we know from the literature that growth conditions such as media, $\mathrm{pH}$, humidity and temperature also affect MVOC production [33]-[35]. More than 200 volatile compounds have been reported as fungi MVOCs in the literature. The combination of large number and variable MVOC composition requires multivariate analysis for specific fungal isolate identification.

Based on standard VOCs absorption data, the CAR/PDMS SPME fiber was considered to be the best fiber for A. flavus VOCs profiling. The time course experiments (carried out over 30 days) revealed that MVOCs production is time-dependent and that aflatoxigenic and non-aflatoxigenic strains had significantly different MVOCs expression patterns. HS-SPME-GCMS was applied successfully to detect and differentiate two A. flavus strains (aflatoxigenic and non-aflatoxigenic strains). A discriminate analysis plot achieved satisfactory performance in classifying A. flavus strains and control based on quantitative MVOCs data even though different isolates produce similar MVOCs. Results indicate that it is possible to build a database for chemotaxonomic application by performing MVOC monitoring at controlled growth conditions (temperature, humidity and substrate). Our sample size is small but clearly shows that specific MVOCs are unlikely to be useful for the confident identification of different A. flavus isolates. Future studies will be done to expand the number of fungal strains that can be dis- 
criminated using patterns of MVOCs instead of individual MVOCs that have been identified with HS-SPMEGCMS using multivariate analysis in order to build up a fungi screening database.

\section{Acknowledgements}

The authors thank the Mississippi Corn Promotion Board for partial funding of this research and Mary Scruggs for excellent technical assistance. This article reports the results of research only. Mention of trade names or commercial products is solely for the purpose of providing specific information and does not imply recommendation or endorsement by Mississippi State University.

\section{References}

[1] Diener, U.L., Cole, R.J., Sanders, T.H., Payne, G.A., Lee, L.S. and Klich, M.A. (1987) Epidemiology of Aflatoxin Formation by Aspergillus flavus*. Annual Review of Phytopathology, 25, 249-270. http://dx.doi.org/10.1146/annurev.py.25.090187.001341

[2] Kurtzman, C., Horn, B. and Hesseltine, C. (1987) Aspergillus nomius, a New Aflatoxin-Producing Species Related to Aspergillus flavus and Aspergillus tamarii. Antonie van Leeuwenhoek, 53, 147-158. http://dx.doi.org/10.1007/BF00393843

[3] Yu, J., Chang, P.K., Cary, J.W., Wright, M., Bhatnagar, D., Cleveland, T.E., Payne, G.A. and Linz, J.E. (1995) Comparative Mapping of Aflatoxin Pathway Gene Clusters in Aspergillus parasiticus and Aspergillus flavus. Applied and Environmental Microbiology, 61, 2365-2371.

[4] Gourama, H. and Bullerman, L.B. (1995) Aspergillus flavus and Aspergillus parasiticus: Aflatoxigenic Fungi of Concern in Foods and Feeds: A Review. Journal of Food Protection, 58, 1395-1404.

[5] Wu, F. and Guclu, H. (2012) Aflatoxin Regulations in a Network of Global Maize Trade. PLoS ONE, 7, Article ID: e45151. http://dx.doi.org/10.1371/journal.pone.0045151

[6] Egmond, H., Schothorst, R. and Jonker, M. (2007) Regulations Relating to Mycotoxins in Food. Analytical and Bioanalytical Chemistry, 389, 147-157. http://dx.doi.org/10.1007/s00216-007-1317-9

[7] van Egmond, H.P., Jonker, M.A. and Abbas, H. (2005) Worldwide Regulations on Aflatoxins. Aflatoxin and Food Safety, 77-93.

[8] Bhatnagar, D., Cary, J.W., Ehrlich, K., Yu, J. and Cleveland, T.E. (2006) Understanding the Genetics of Regulation of Aflatoxin Production and Aspergillus flavus Development. Mycopathologia, 162, 155-166. http://dx.doi.org/10.1007/s11046-006-0050-9

[9] Turner, N.W., Subrahmanyam, S. and Piletsky, S.A. (2009) Analytical Methods for Determination of Mycotoxins: A Review. Analytica Chimica Acta, 632, 168-180. http://dx.doi.org/10.1016/j.aca.2008.11.010

[10] Tsui, C.K., Woodhall, J., Chen, W., Levesque, C.A., Lau, A., Schoen, C.D., Baschien, C., Najafzadeh, M.J. and de Hoog, G.S. (2011) Molecular Techniques for Pathogen Identification and Fungus Detection in the Environment. IMA Fungus, 2, 177-189. http://dx.doi.org/10.5598/imafungus.2011.02.02.09

[11] Korpi, A., Jarnberg, J. and Pasanen, A.L. (2009) Microbial Volatile Organic Compounds. Critical Reviews in Toxicology, 39, 139-193. http://dx.doi.org/10.1080/10408440802291497

[12] Schnürer, J., Olsson, J. and Börjesson, T. (1999) Fungal Volatiles as Indicators of Food and Feeds Spoilage. Fungal Genetics and Biology, 27, 209-217. http://dx.doi.org/10.1006/fgbi.1999.1139

[13] Sunesson, A., Vaes, W., Nilsson, C., Blomquist, G., Andersson, B. and Carlson, R. (1995) Identification of Volatile Metabolites from Five Fungal Species Cultivated on Two Media. Applied and Environmental Microbiology, 61, 2911-2918.

[14] Keshri, G., Magan, N. and Voysey, P. (1998) Use of an Electronic Nose for the Early Detection and Differentiation between Spoilage Fungi. Letters in Applied Microbiology, 27, 261-264. http://dx.doi.org/10.1046/j.1472-765X.1998.00438.x

[15] Nilsson, T., Larsen, T.O., Montanarella, L. and Madsen, J.Ø. (1996) Application of Head-Space Solid-Phase Microextraction for the Analysis of Volatile Metabolites Emitted by Penicillium Species. Journal of Microbiological Methods, 25, 245-255. http://dx.doi.org/10.1016/0167-7012(95)00093-3

[16] Jurjevic, Z., Rains, G.C., Wilson, D.M. and Lewis, W.J. (2009) Volatile Metabolites Associated with One Aflatoxigenic and One Nontoxigenic Aspergillus flavus Strain Grown on Two Different Substrates. Phytopathologia Mediterranea, 47, 266-271.

[17] Schleibinger, H., Laußmann, D., Brattig, C., Mangler, M., Eis, D. and Ruden, H. (2005) Emission Patterns and Emission Rates of MVOC and the Possibility for Predicting Hidden Mold Damage? Indoor Air, 15, 98-104. 
http://dx.doi.org/10.1111/j.1600-0668.2005.00349.x

[18] Thorn, R.M., Reynolds, D.M. and Greenman, J. (2011) Multivariate Analysis of Bacterial Volatile Compound Profiles for Discrimination between Selected Species and Strains in Vitro. Journal of Microbiological Methods, 84, 258-264. http://dx.doi.org/10.1016/j.mimet.2010.12.001

[19] Bianchi, F., Careri, M., Mangia, A., Mattarozzi, M., Musci, M., Concina, I., Falasconi, M., Gobbi, E., Pardo, M. and Sberveglieri, G. (2009) Differentiation of the Volatile Profile of Microbiologically Contaminated Canned Tomatoes by Dynamic Headspace Extraction Followed by Gas Chromatography-Mass Spectrometry Analysis. Talanta, 77, 962-970. http://dx.doi.org/10.1016/j.talanta.2008.07.061

[20] Larsen, T.O. and Frisvad, J.C. (1995) Comparison of Different Methods for Collection of Volatile Chemical Markers from Fungi. Journal of Microbiological Methods, 24, 135-144. http://dx.doi.org/10.1016/0167-7012(95)00063-1

[21] Demyttenaere, J.C.R., Moriña, R.M. and Sandra, P. (2003) Monitoring and Fast Detection of Mycotoxin-Producing Fungi Based on Headspace Solid-Phase Microextraction and Headspace Sorptive Extraction of the Volatile Metabolites. Journal of Chromatography A, 985, 127-135. http://dx.doi.org/10.1016/S0021-9673(02)01417-6

[22] Lavine, B.K., Mirjankar, N., LeBouf, R. and Rossner, A. (2012) Prediction of Mold Contamination from Microbial Volatile Organic Compound Profiles Using Solid Phase Microextraction and Gas Chromatography/Mass Spectrometry. Microchemical Journal, 103, 37-41. http://dx.doi.org/10.1016/j.microc.2012.01.002

[23] Vishwanath, V., Sulyok, M., Weingart, G., Kluger, B., Täubel, M., Mayer, S., Schuhmacher, R. and Krska, R. (2011) Evaluation of Settled Floor Dust for the Presence of Microbial Metabolites and Volatile Anthropogenic Chemicals in Indoor Environments by LC-MS/MS and GC-MS Methods. Talanta, 85, 2027-2038. http://dx.doi.org/10.1016/j.talanta.2011.07.043

[24] Drew, D.P., Rasmussen, S.K., Avato, P. and Simonsen, H.T. (2012) A Comparison of Headspace Solid-Phase Microextraction and Classic Hydrodistillation for the Identification of Volatile Constituents from Thapsia spp. Provides Insights into Guaianolide Biosynthesis in Apiaceae. Phytochemical Analysis, 23, 44-51. http://dx.doi.org/10.1002/pca.1323

[25] Gioacchini, A.M., Menotta, M., Bertini, L., Rossi, I., Zeppa, S., Zambonelli, A., Piccoli, G. and Stocchi, V. (2005) Solid-Phase Microextraction Gas Chromatography/Mass Spectrometry: A New Method for Species Identification of Truffles. Rapid Communications in Mass Spectrometry, 19, 2365-2370. http://dx.doi.org/10.1002/rcm.2031

[26] Siripatrawan, U. and Harte, B.R. (2007) Solid Phase Microextraction/Gas Chromatography/Mass Spectrometry Integrated with Chemometrics for Detection of Salmonella typhimurium Contamination in a Packaged Fresh Vegetable. Analytica Chimica Acta, 581, 63-70. http://dx.doi.org/10.1016/j.aca.2006.08.007

[27] Siripatrawan, U. (2008) Rapid Differentiation between E. coli and Salmonella Typhimurium Using Metal Oxide Sensors Integrated with Pattern Recognition. Sensors and Actuators B: Chemical, 133, 414-419. http://dx.doi.org/10.1016/j.snb.2008.02.046

[28] Demain, A. (1986) Regulation of Secondary Metabolism in Fungi. Pure and Applied Chemistry, 58, 219-226. http://dx.doi.org/10.1351/pac198658020219

[29] Augusto, F. and Valente, A.L.P. (2002) Applications of Solid-Phase Microextraction to Chemical Analysis of Live Biological Samples. TrAC Trends in Analytical Chemistry, 21, 428-438. http://dx.doi.org/10.1016/S0165-9936(02)00602-7

[30] Zeringue, H.J., Bhatnagar, D. and Cleveland, T.E. (1993) $\mathrm{C}_{15} \mathrm{H}_{24}$ Volatile Compounds Unique to Aflatoxigenic Strains of Aspergillus flavus. Applied and Environmental Microbiology, 59, 2264-2270.

[31] Payne, G.A. and Brown, M.P. (1998) Genetics and Physiology of Aflatoxin Biosynthesis. Annual Review of Phytopathology, 36, 329-362. http://dx.doi.org/10.1146/annurev.phyto.36.1.329

[32] Börjesson, T., Stöllman, U. and Schnürer, J. (1992) Volatile Metabolites Produced by Six Fungal Species Compared with Other Indicators of Fungal Growth on Cereal Grains. Applied and Environmental Microbiology, 58, 2599-2605.

[33] Larsen, T.O. and Frisvad, J.C. (1994) Production of Volatiles and Presence of Mycotoxins in Conidia of Common Indoor Penicillia and Aspergilli. Elsevier Science, Amsterdam, 251-279.

[34] Batterman, S.A. (1995) Sampling and Analysis of Biological Volatile Organic Compounds. Bioaerosols, CRC Press Inc., Boca Raton, 249-268.

[35] Korpi, A., Pasanen, A.L. and Pasanen, P. (1998) Volatile Compounds Originating from Mixed Microbial Cultures on Building Materials under Various Humidity Conditions. Applied and Environmental Microbiology, 64, 2914-2919. 